\title{
Transient Silencing of a Type IV P-Type ATPase, Atp10c, Results in Decreased Glucose Uptake in C2C12 Myotubes
}

\author{
S. E. Hurst, ${ }^{1,2}$ S. C. Minkin, ${ }^{3}$ J. Biggerstaff, ${ }^{3}$ and M. S. Dhar ${ }^{2}$ \\ ${ }^{1}$ Comparative and Experimental Medicine, College of Veterinary Medicine, University of Tennessee, Knoxville, TN 37996, USA \\ ${ }^{2}$ Department of Large Animal Clinical Sciences, College of Veterinary Medicine, University of Tennessee, Knoxville, TN 37996, USA \\ ${ }^{3}$ Center for Environmental Biotechnology, University of Tennessee, Knoxville, TN 37996, USA
}

Correspondence should be addressed to S. E. Hurst, shurst6@utk.edu

Received 15 August 2011; Revised 15 October 2011; Accepted 29 October 2011

Academic Editor: Duo Li

Copyright () 2012 S. E. Hurst et al. This is an open access article distributed under the Creative Commons Attribution License, which permits unrestricted use, distribution, and reproduction in any medium, provided the original work is properly cited.

\begin{abstract}
Atp10c is a strong candidate gene for diet-induced obesity and type 2 diabetes. To identify molecular and cellular targets of ATP10C, Atp10c expression was altered in vitro in C2C12 skeletal muscle myotubes by transient transfection with an Atp10cspecific siRNA. Glucose uptake assays revealed that insulin stimulation caused a significant 2.54-fold decrease in 2-deoxyglucose uptake in transfected cells coupled with a significant upregulation of native mitogen-activated protein kinases (MAPKs), p38, and p44/42. Additionally, glucose transporter-1 (GLUT1) was significantly upregulated; no changes in glucose transporter-4 (GLUT4) expression were observed. The involvement of MAPKs was confirmed using the specific inhibitor SB203580, which downregulated the expression of native and phosphorylated MAPK proteins in transfected cells without any changes in insulin-stimulated glucose uptake. Results indicate that Atp10c regulates glucose metabolism, at least in part via the MAPK pathway, and, thus, plays a significant role in the development of insulin resistance and type 2 diabetes.
\end{abstract}

\section{Introduction}

In humans, skeletal muscle accounts for nearly $40 \%$ of the body's mass and serves as the main tissue involved in glucose uptake during insulin stimulation. Several researchers have established that glucose consumption in skeletal muscle decreases with type 2 diabetes mellitus. This reduced glucose consumption is a result of impaired transduction of insulin signals, such as insulin receptor substrate-1 phosphorylation, phosphatidylinositol 3-kinase (PI3K) activity, mitogen-activated protein kinase (MAPK) activity, insulinresponsive glucose transporters, namely, glucose transporter4 (GLUT4), and/or other insulin-independent mechanisms [1]. Therefore, a detailed analysis of insulin signaling at the cellular and molecular level is critical to understand the pathogenesis of type 2 diabetes associated with obesity.

Heterozygous Atp10c mice present with the disease states of insulin resistance and obesity, as well as a host of other related disorders, including hyperlipidemia and hyperinsulinemia. Previous research using these mice indicates that the Atp10c gene appears to be a strong candidate gene for diet-induced obesity and type 2 diabetes mellitus [2]. Atp10c is a putative phospholipid translocase or "flippase," which encodes for a type IV P-type ATPase. Atp10c maps to the $p$-locus on mouse chromosome 7 , to a region of a quantitative trait locus associated with body weight, body fat, and diabetic phenotypes. The human ortholog, ATP10C, maps to the syntenic region on chromosome $15 q 12$ and is also associated with an elevated body mass index $[3,4]$. Moreover, microarray gene profiling on Atp10c heterozygous mice indicated significant changes in the mRNA expression of factors involved in insulin-dependent and insulinindependent glucose uptake [5].

Although flippases, like Atp10c, have been studied for many years, their exact character and function remain unclear. These proteins are believed to maintain the asymmetry of the lipid bilayer by translocating specific phospholipids from one leaflet to the other and vice versa [6], but they may also participate in the formation of transport vesicles [7]. Moreover, deficiencies in these proteins have been shown to cause defects in lipid metabolism and have been implicated in the disease states of obesity, type 2 diabetes, 
and nonalcoholic fatty liver disease [2]. Not much is known about the role of ATP10C in regulating insulin resistance in skeletal muscle, if any, and its possible molecular and cellular targets have not been investigated.

In view of the above literature, we hypothesized that the type IV P-type ATPase, ATP10C, has an important role in glucose metabolism. Since ATP10C is a transmembrane protein it might exert its effect via multiple signaling pathways: (1) acting solely at the plasma membrane to maintain the nonrandom distribution of phospholipids, thus contributing to a proper membrane environment for normal protein sequestration and function, (2) acting at the plasma membrane affecting the biogenesis of membrane vesicles important for plasma membrane delivery and/or retrieval of glucose transporter proteins in basal and insulin-stimulated states, and (3) acting directly on the expression, translocation, and/or function of glucose transporter proteins themselves. Since the MAPK pathway is known to be a key signaling cascade which mediates glucose clearance/uptake by the skeletal muscle in presence or absence of insulin, we tested whether the MAPKs, in general, are the targets of ATP10C. To prove our hypothesis, specific objectives were to (a) establish a tissue culture system of mouse skeletal muscle wherein endogenous expression of Atp10c could be monitored, (b) alter the endogenous level of Atp10c expression by siRNA, and (c) measure glucose uptake and assess changes in expression of MAPKs involved in this process.

\section{Materials and Methods}

2.1. Materials. Mouse skeletal muscle cell line C2C12, a commercially available cell line, was kindly provided by Dr. Seung Baek, College of Veterinary Medicine, the University of Tennessee, Knoxville, TN, USA. Dulbecco's modified Eagle medium (DMEM) containing $4.5 \mathrm{mg} / \mathrm{L}$ glucose and $4.5 \mathrm{mM} / \mathrm{L}$ L-glutamine, antibiotics $(100 \mathrm{IU} / \mathrm{mL}$ penicillin and $100 \mu \mathrm{g} / \mathrm{mL}$ streptomycin), ABsolute Blue SYBR Green ROX quantitative PCR mix, bovine calf serum (BCS), and radioimmunoprecipitation assay (RIPA) buffer were from Thermo Fisher Scientific (Waltham, MA). DMEM with 1\% antibiotics and $10 \%$ BCS is furthermore referred to as the complete growth media. Horse serum, 2-deoxy $\left[{ }^{3} \mathrm{H}\right]$ glucose (2-DOG), protease inhibitor cocktail in DMSO solution, MAPK inhibitor SB203580, and human insulin solution $(10 \mathrm{mg} / \mathrm{mL}$ in HEPES, $\mathrm{pH} 8.2)$ were from Sigma Aldrich (St. Louis, MO). A bicinchoninic acid kit (BCA) and an enhanced chemiluminescence (ECL) western blotting Detection Kit were purchased from Pierce Biotech Inc. (Rockford, IL) and used in protein experiments. Primary antibodies (p38, phospho-p38, JNK, phospho-JNK, p44/42, and phospho-p44/42) as well as the secondary antibody, horseradish peroxidase- (HRP-) conjugated anti-rabbit IgG, were obtained from Cell Signaling Technology (Danvers, MA). Caveolin-1 was used as an immunoblot control and was purchased from Santa Cruz Biotechnologies (Santa
Cruz, CA). The secondary antibody, HRP-conjugated antigoat IgG, was also obtained from Santa Cruz Biotechnologies. HiPerfect transfection reagent, Atp10c-specific siRNA constructs, RNeasy Mini Kit, and QuantiTect primer assays for $M y o D$ and Atp10c were from Qiagen (Valencia, CA). Quantitative PCR primers specific for mouse glyceraldehyde 3-phosphate dehydrogenase (Gapdh) were designed using the Primer 3 program (http://primer3.sourceforge.net/) and were commercially obtained from Operon (Huntsville, AL). The iScript cDNA synthesis kit was acquired from Bio-Rad Laboratories (Hercules, CA). Anti-GLUT1 and GLUT4 were kindly provided by Dr. Samuel Cushman, National Institute of Diabetes and Digestive and Kidney Diseases, National Institutes of Health, Bethesda, MD. All immunofluorescence materials (protein blocks [normal rabbit], negative control [normal rabbit], and antibody diluent) were purchased from BioGenex (San Ramon, CA). Millicell EZ slides from Millipore (Billerica, MA) were used for immunofluorescence experiments. Secondary antibody specific for immunofluorescence application, Alexa Fluor 568 donkey anti-rabbit, as well as Prolong Gold Antifade reagent was purchased from Invitrogen (Carlsbad, CA).

2.2. Cell Culture and Treatments. C2C12 myoblasts were cultured as described elsewhere $[8,9]$. Roughly $2.0 \times 10^{5}$ cells were seeded in a $60 \mathrm{~mm}$ dish or a single well of a 6 -well plate. They were maintained at $37^{\circ} \mathrm{C}$ and $5 \% \mathrm{CO}_{2}$ in complete growth media. Cells at $70 \%$ confluency were differentiated in the presence of $2 \%$ horse serum-enriched media for 3-5 days. Completely differentiated myotubes (days 3-5) were either subjected to various treatments described in the relevant sections or harvested for subsequent experiments.

2.3. siRNA Transfection. Three different siRNA oligonucleotides against Atp10c were commercially obtained (Qiagen); one was generated from the sequence at the $3^{\prime}$ end of the Atp10c gene, SI00906220 (sense: r[CCU GGG UAU UGA AAC CAA A]dTdT and antisense: r[UUU GGU UUC AAU ACC CAG G]dTdG), and the second, SI00906213, and third, SI00906206, were generated from the sequence at the $5^{\prime}$ end (sense: r[CGU CUU UGC UGC AAU GAA A]dTdT and antisense: $r$ [UUU CAU UGC AGC AAA GAC G]dGdA). C2C12 myotubes were transiently transfected with Atp10c-specific and scrambled siRNA using HiPerfect transfection reagent (Qiagen) according to the manufacturer's instructions. Myotubes transfected with siRNA were either harvested or treated with the reagents indicated in the relevant sections and/or used to measure glucose uptake.

In the first experiment, the optimum concentration and time of knockdown for each siRNA used were determined. Briefly, HiPerfect transfection regent and siRNAs were mixed at various concentrations $(0,50,100$, and $200 \mathrm{nM})$ to form a complex. The transfection complexes were then applied to designated cells and incubated for 24,48 , or $72 \mathrm{~h}$ before subsequent analysis. C2C12 myotubes demonstrating efficient Atp10c knockdown and, therefore, used in all further experiments were designated as $\mathrm{C} 210 \mathrm{c} /-$. Mock-transfected 
(i.e., transfected with HiPerfect only) $\mathrm{C} 2 \mathrm{C} 12$ myotubes (C2wt) were used as corresponding controls.

2.4. RNA, cDNA Synthesis, and Quantitative PCR. The following procedures were performed as described elsewhere $[5,10,11]$. Cells were washed with ice cold $1 \mathrm{X}$ PBS, and total RNAs were isolated using RNeasy Mini RNA kit (Qiagen), according to the manufacturer's instructions. Single-stranded cDNA was synthesized using the iScript cDNA synthesis kit (Bio-Rad) and amplified using genespecific primers by quantitative PCR (qPCR by Quantitect primer assays), with mouse Gapdh as the housekeeping gene. All mRNA expressions were achieved by qPCR using ABsolute SYBR Green ROX quantitative PCR mix on the Strategene Mx3005P with MxPro analysis software under the following PCR conditions: 1 cycle of $50^{\circ} \mathrm{C}$ for $15 \mathrm{~min}$ and $95^{\circ} \mathrm{C}$ for $2 \mathrm{~min}$, followed by 40 cycles of $95^{\circ} \mathrm{C}$ for $25 \mathrm{~s}$, $52^{\circ} \mathrm{C}$ for $25 \mathrm{~s}$, and $72^{\circ} \mathrm{C}$ for $1 \mathrm{~min}$. The relative abundance of target gene expression was calculated using the 2- $\Delta \Delta \mathrm{CT}$ and standard curve method, with $\Delta \Delta \mathrm{CT}$ being the difference between $\mathrm{CT}$ of the target gene normalized with respect to the Gapdh CT [12].

2.5. Preparation of Cellular Extracts, Immunoblotting, and Immunofluorescence. Total cell lysates were isolated using RIPA buffer according to standard methods [13, 14]. Briefly, cells were washed twice with 1X PBS and lysed in RIPA buffer containing protease inhibitor cocktails at $4^{\circ} \mathrm{C}$ for $30 \mathrm{~min}$. Lysates were centrifuged at $16,000 \mathrm{~g}$ for $10 \mathrm{~min}$ at $4^{\circ} \mathrm{C}$. Protein estimation was performed using the BCA kit (Pierce Biotech), according to the manufacturer's instructions.

Immunoblot analysis was carried out according to standard procedures $[13,14]$. Equal concentrations $(25-100 \mu \mathrm{gs})$ of proteins were resolved on 10\% SDS-PAGE, using 5X Laemmli sample buffer containing Tris-HCL $(375 \mathrm{mM}$, pH 6.8), glycerol (48\%), SDS (6\%), beta-mercaptoethanol (6\%), and bromophenol $(0.03 \%)$. Cell lysates were denatured by heating before being applied to SDS-PAGE gel. After electrophoresis, proteins were transferred to nitrocellulose membranes, blocked for $1 \mathrm{~h}$ in blocking solution (1$5 \%$ BSA in TBST buffer), and incubated with specific primary antibodies overnight at $4^{\circ} \mathrm{C}$. Primary antibodies were detected with HRP-conjugated secondary antibodies, and antibody-protein complexes were visualized using ECL (Pierce Biotech). Results are expressed as the ratio of target protein expression to that of an internal loading control, caveolin-1.

For immunofluorescence, $2.0 \times 10^{4}$ cells were seeded onto 4-well chamber slides (Millipore) and subjected to the appropriate treatments as described in the relevant sections. Immunofluorescence assays were carried out according to standard methods as described by others $[8,15]$. Briefly, cells were fixed with $1 \%$ paraformaldehyde in $0.1 \mathrm{M}$ sodium phosphate buffer, $\mathrm{pH} 7.3$ for $10 \mathrm{~min}$ at room temperature. Cells were washed in 1X PBS, permeabilized by incubating with $0.01 \%$ Tween-20/PBS for $10 \mathrm{~min}$, and then washed again with $1 \mathrm{X}$ PBS. After the last wash, cells were blocked using blocking buffer (1\% BSA, $2 \%$ normal serum, $0.1 \%$
Tween-20 in PBS) for 30 min. Blocking solution contained normal rabbit serum, the animal in which the primary antibody was generated. Once blocking was complete, the cells were incubated with specific primary antibodies overnight at $4^{\circ} \mathrm{C}$. Bound antibody was visualized under the microscope (Nikon Ti-E Eclipse, Nikon Instruments, Melville, NY) by incubating for $1 \mathrm{~h}$ with secondary antibody labeled with Alexa Fluor 568 (TRITC). To visualize the nucleus, cells were exposed for $5 \mathrm{~min}$ at room temperature to a concentration of $300 \mathrm{nM}$ DAPI (4', 6-diamidino-2phenylindole, dilactate) in PBS. DAPI was prepared and diluted based on manufacturer's instructions (Invitrogen). After washing, cells were mounted using ProLong Gold Antifade reagent (Invitrogen). Slides were sealed and allowed to dry overnight before imaging. Additionally, both positive and negative controls were prepared and imaged alongside the samples to correct for any background fluorescence and to serve as controls for quantitative analysis. Images were captured using an epifluorescence microscope (Nikon Instruments) with a 60x objective lens (NA 1.49) and an automated stage.

2.6. Glucose Uptake. Glucose uptake in myotubes was measured as previously described [11]. Briefly, myoblasts were plated in 6-well cell culture plates at a density of $2 \times$ $10^{5}$ cells/well and allowed to differentiate under normal conditions. After differentiation, cells were washed with IX PBS and serum-starved in DMEM only for $3-5 \mathrm{~h}$. Cells were stimulated with $100 \mathrm{nM}$ insulin in DMEM for $30 \mathrm{~min}$ at $37^{\circ} \mathrm{C}$. Each 6-well plate was setup so that wells 1, 2, and 3 did not contain insulin and wells 4, 5, and 6 did contain insulin. Insulin induction was stopped by washing the cells twice with $1 \mathrm{~mL}$ Krebs-Ringer HEPES (KRP) $(121 \mathrm{mM}$ $\mathrm{NaCl}, 4.9 \mathrm{mM} \mathrm{KCl}, 1.2 \mathrm{mM} \mathrm{MgSO}_{4}, 0.33 \mathrm{mM} \mathrm{CaCl}_{2}, 12 \mathrm{mM}$ HEPES) minus glucose at room temperature. Cytochalasin $\mathrm{B}$ ( $5 \mu \mathrm{L}$ of $1 \mathrm{mM}$ stock $/ 1 \mathrm{~mL}$ cocktail) was used to normalize for nonspecific glucose uptake. Glucose uptake was determined after the addition of ${ }^{3} \mathrm{H}$-2-deoxyglucose $(1 \mu \mathrm{L}$ of $10 \mathrm{Ci} / \mathrm{mmol}$ stock $/ 1 \mathrm{~mL}$ cocktail) in KRP buffer at $37^{\circ} \mathrm{C}$ for $5 \mathrm{~min}$. Incorporation was terminated by washing the cells twice with $1 \mathrm{~mL}$ ice cold KRP plus $25 \mathrm{mM}$ glucose. Cells were lysed on ice for 30 min with RIPA buffer. Following incubation, $0.5 \mathrm{~mL}$ cell lysates were mixed with $10 \mathrm{~mL}$ scintillation fluid (Scintiverse) and subjected to liquid scintillation counting. For protein quantitation (BCA method, Pierce Biotech), $250 \mu \mathrm{L}$ of the lysate was used and processed according to the manufacturer's instructions. Glucose uptake was expressed as disintegrations per minute per microgram of total protein $(\mathrm{dpm} / \mu \mathrm{g})$. Data is reported as the glucose uptake stimulation expressed as the ratio of $\mathrm{dpm} / \mu \mathrm{g}$ of total protein in presence of insulin to that in the absence of insulin.

2.7. Densitometry Analysis. Relative densitometry analyses of the immunoblots were determined using Scion (http://scion-image.software.informer.com/) and Image J (http://rsb.info.nih.gov/ij/index.html) analysis software. By giving an arbitrary value of 1.0 to the respective control 
sample (caveolin-1) of each experiment, a ratio of relative density was calculated for each protein of interest.

2.8. Immunofluorescence Quantitation. Immunofluorescence images were analyzed using NIS-Elements software (AR v. 3.1) (Nikon Instruments). For each sample, 9 dual-channel images were captured and stitched together to form a large image $(3 \times 3)$. Mean fluorescence intensity per cell was calculated (MFI per cell $=$ total image fluorescence/cell count). Cell count was determined using a nuclear stain (300 nM DAPI in PBS).

2.9. Statistical Analysis. The data are expressed as mean \pm SE. For comparison of two groups, $P$ values were calculated using the two-tailed paired Student $t$ test. In all cases $P \leq$ 0.05 was considered statistically significant, and $P \leq 0.10$ was indicative of a trend.

\section{Results and Discussion}

To alleviate whole-animal complexities, $\mathrm{C} 2 \mathrm{C} 12$, a mouse skeletal muscle cell line, was selected as an in vitro model to identify molecular and cellular targets of ATP10C and then assess its biological role, if any, in insulin signaling and glucose metabolism [16, 17].

Under permissive conditions, $\mathrm{C} 2 \mathrm{C} 12$ myoblasts undergo differentiation to form myotubes. Differentiation of C2C12 myoblasts to myotubes was confirmed by visual inspection (see Supplemental Figure 1 in Supplementary Material available online at doi.10.1155/2012/152902), as well as by monitoring the expression of skeletal muscle-specific mRNAs for MyoD and myogenin [18]. Cells were collected on days 1,3 , and 5 during the differentiation process. Cells collected at day 1 represent the myoblasts, which are then stimulated to differentiate into myotubes by days 3-5. The activation of cell differentiation is characterized by the expression of myogenic regulatory factors including $M y o D$ and myogenin. After proliferation, cells express myogenin which commits the myoblasts towards myogenic differentiation. This is followed by the expression of additional factors including but not limited to Myf-5 and MRF4 and permanent exit from the normal growth and proliferation cycles. As expected, the expression of myogenin increased as myoblasts were stimulated to differentiate (30\% to $52 \%$ ), and decreased when differentiation was complete $(20 \%)$. Similarly, qPCR showed that $M y o D$ expression increased dramatically on day $1(172 \%)$, then steadily declined on days 3 and 5 (64\% and 24\%, resp.). Interestingly, Atp10c was expressed in both cell types, and its expression increased upon differentiation ( $42 \%$ to $64 \%)$ and steadily decreased as myotubes were formed $(47 \%$ on day 5$)$. Changes in Atp10c expression with differentiation were similar, but, not as striking as that of myogenin and $M y o D$ (Supplemental Figure 2), ramifications of which are clearly beyond the scope of this study. The data thus demonstrated the expression of Atp10c mRNA in $\mathrm{C} 2 \mathrm{C} 12$ cells, and gave us an opportunity to modulate its expression in both myoblasts and myotubes,

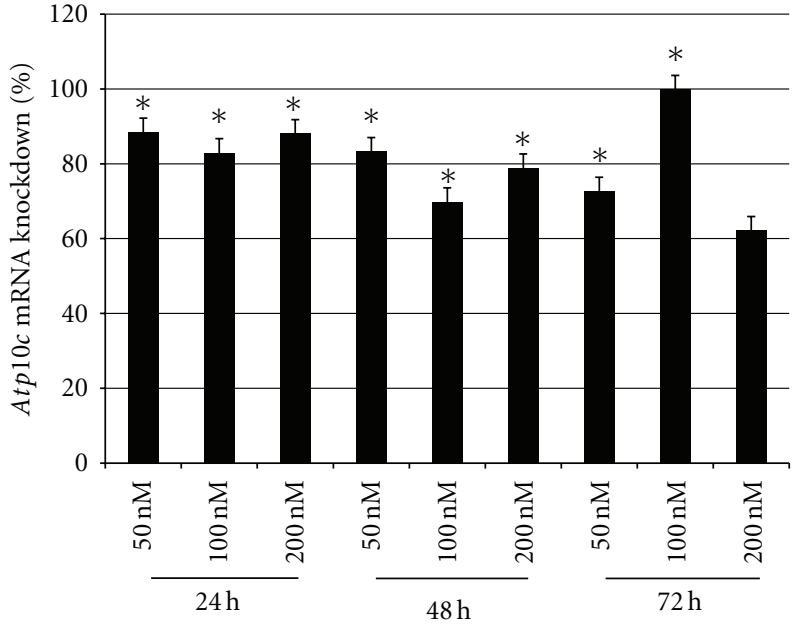

FIgURE 1: C2C12 cells were differentiated from myoblasts to myotubes as described in Section 2. Myotubes were transfected at each concentration of siRNA (SI00906220) $(0,50,100$, and $200 \mathrm{nM})$ collected at the above time points (24, 48, and $72 \mathrm{~h}$ ). Gapdh (housekeeping gene) and Atp10c gene mRNA expression was analyzed using quantitative PCR. The percentage of knockdown was calculated at each concentration and time point based on the expression of the mock-transfected $(0 \mathrm{nM})$ samples $\left({ }^{*} P<0.7\right.$ based on Qiagen recommendations). Data represents three independent experiments with each sample repeated in triplicate.

both of which can have significant consequences in insulin signaling pathways.

One of the limitations of global gene-targeting and whole-animal approach is that adaptations over time might occur, possibly producing secondary phenotypes that are not directly linked to the mutation. In this case, the role of Atp10c could be shown either by generating knockout mice or using specific inhibitors against ATP10C. Since no inhibitors are available, and to avoid whole-animal complexities in transgenics, we modulated Atp10c expression in vitro in $\mathrm{C} 2 \mathrm{C} 12$ cells by transiently transfecting them with three independent and commercially available Atp $10 c$-specific siRNAs (Qiagen). ATP10C is a putative transmembrane domain protein, and as such a good antibody against ATP10C has yet to be generated, making experiments to study ATP10C challenging. Therefore, in this analysis, changes in Atp10c expression were determined solely at the mRNA level by quantitative PCR using QuantiTect primer assays (Qiagen). Out of the three siRNAs tested, only one, SI00906220, resulted in a significant knockdown of $\geq 70 \%$ (data not shown).

As shown in Figure 1, significant knockdowns (70 to $100 \%$ ) were observed in all samples with the exception of $200 \mathrm{nM}, 72 \mathrm{~h}$. Higher concentrations and longer time periods resulted in cell death and poor quality of cells (as judged visually). Taking these criteria into consideration as well as the optimization of concentration and time of transfection, $50 \mathrm{nM}$ of siRNA (SI00906220) and a time point of $24 \mathrm{~h}$ were selected for all subsequent experiments. Gapdh was used as the housekeeping gene, and no significant mRNA 


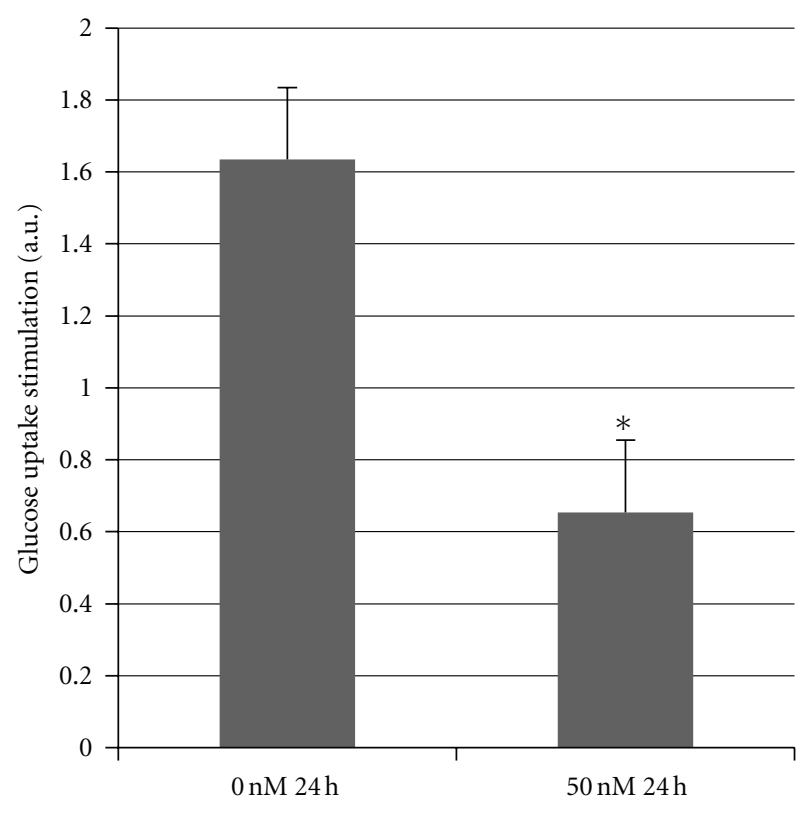

Figure 2: C2C12 cells were differentiated from myoblasts to myotubes as described in Section 2. Myotubes were transfected at each concentration of siRNA (SI00906220) $(0$ and $50 \mathrm{nM})$ at the designated time point $(24 \mathrm{~h})$. Cells were then stimulated with insulin $(100 \mathrm{nM}, 30 \mathrm{~min})$, and a 2-DOG uptake was performed $\left({ }^{*} P<0.05\right)$. Data is reported as the glucose uptake stimulation which is expressed as the ratio of $\mathrm{dpm} / \mu \mathrm{g}$ of total protein in presence of insulin to that in the absence of insulin.

changes in its corresponding expression in transfected cells were observed. Results thus suggest that changes in Atp10c expression after transfection with siRNA (SI00906220) were not an artifact, and there was no deleterious effect on $\mathrm{C} 2 \mathrm{C} 12$ myotubes due to Atp10c silencing. All further experiments were carried out under these conditions in wildtype $\mathrm{C} 2 \mathrm{C} 12(\mathrm{C} 2 \mathrm{wt})$ and Atp10c-silenced $\mathrm{C} 2 \mathrm{C} 12$ myotubes $(\mathrm{C} 210 c /-)$ simultaneously. Since these transfections are transient, Atp10c mRNA knockdown was confirmed in each and every experiment.

Previous experiments in our laboratory have shown that on a high fat diet, there is a $35 \%$ decrease in glucose uptake in soleus muscle in Atp10c heterozygotes [5]. Based on this finding, we next determined whether downregulation of Atp10c expression had a similar effect in vitro. Glucose uptake was measured in $\mathrm{C} 2 \mathrm{wt}$ and $\mathrm{C} 210 \mathrm{c} /$ - myotubes in basal (without insulin) and stimulated (100 nM insulin, $30 \mathrm{~min}$ ) states. Fold change representing the glucose uptake stimulation/reduction between the $\mathrm{C} 2 \mathrm{wt}$ and $\mathrm{C} 210 \mathrm{c} /-$ was compared. As shown in Figure 2, insulin stimulation caused a significant 2.54-fold decrease in 2-DOG uptake in C210c/cells $(P<0.05)$. Data thus complement the in vivo findings, suggesting that ATP10C is necessary for insulin-stimulated glucose uptake in skeletal muscle, and its knockdown renders the myotubes insulin resistant.

Silencing Atp10c RNA decreases cellular glucose uptake, which might be of consequence to impaired insulin signaling. Insulin-induced glucose uptake into muscle and adipose tissue involves a series of intracellular signaling cascades, culminating in glucose disposal and metabolism [19-24]. The possible mechanisms include insulin-mediated activation of the insulin receptor and/or its downstream molecules, ultimately effecting GLUT4 expression and translocation. Since these processes are a complex interplay of a variety of proteins and because their changes have not been studied in Atp10c silencing, we sought to identify changes in the key proteins of one such signaling cascade, the MAPK pathway in the absence of any insulin stimulation. Specifically, in the present study, the effect of Atp10c silencing was considered on three essential MAPKs: p38 (MapK14), JNK, and p44/42, (ERK1/2). Total proteins isolated from C2wt and C210c/myotubes were subjected to a combination of immunoblot and immunofluorescence analysis (Figures 3(a)-3(c)).

For MAPK pathway analysis, results indicated significant upregulation of p38 $(P=0.02)$ and $\mathrm{p} 44 / 42(P=0.04)$ and a significant downregulation of JNK $(P=0.001)$ and phospho-p44/42 $(P=0.05)$ in $\mathrm{C} 210 \mathrm{c} /-$ cells. While not significant, results indicated a trend for an increase in phospho-p38 $(P=0.1)$ (Figure 3(a)) and phospho-JNK $(P=0.06)$ (Figure 3(c)). Beside these signal transduction proteins, there was significant upregulation of $\operatorname{MyoD}(P=$ $0.01)$, Actin $(P=0.02)$ (Supplemental Figures 3(a) and $3(\mathrm{~b}))$, and GLUT1 $(P=0.03)$. Most importantly, there was no significant change in GLUT4 expression (Figure 4).

The exact mechanism by which the MAPKs mediate glucose uptake is debatable. This is such a complex process that both in vitro and in vivo data are inconclusive. MAPKs, specifically, p38 and p44/42 proteins, regulate glucose uptake via insulin-dependent and independent pathways [25-27]. Our results suggest that when myotubes become insulin resistant by Atp10c silencing, as expected there is a significant increase in the expression of native MAPKs; however, they are not activated into their phosphorylated forms at a significant level. Data demonstrates that p38 and p44/42 are responsive to changes in Atp10c with increased expression, whereas there is no similar effect on JNK, suggesting that it is not a target at least in this pathway.

The importance of p38 in this process is further supported by the fact that there are significant changes in glucose transporter proteins as well. GLUT1 and GLUT4 have been demonstrated to be the key players of glucose clearance in peripheral tissues [5]. GLUT1 is responsible for glucose uptake in the basal state whereas GLUT4 is insulin responsive [28]. Defective uptake of glucose mediated by the GLUTs is a central feature of obesity and type 2 diabetes. Interestingly, microarray gene profiling of peripheral tissues obtained from Atp10c heterozygotes which were fed a high fat diet for 4 weeks demonstrated upregulation of p38 in soleus muscle [5].

Of relevance to our study, MAPK protein expression, specifically $\mathrm{p} 38$, has been shown to affect the expression of GLUT1 and GLUT4, which can subsequently affect glucose uptake in peripheral tissues [28]. The MAPK protein, p38, reportedly upregulates the expression of GLUT1, thereby altering glucose transport at the basal level, while also involved in an insulin-induced enhancement of intrinsic GLUT4 activity on the cell surface. Since, we do not see 


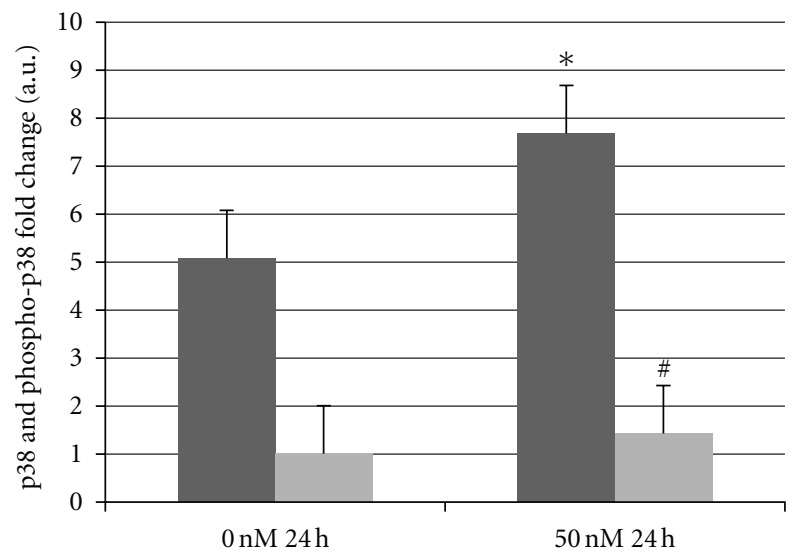

p38

pp38

(a)

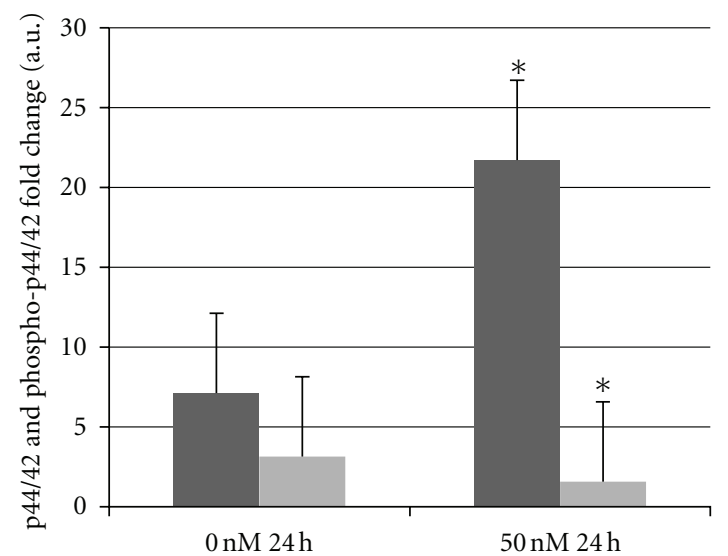

$\mathrm{p} 44 / 42$

pp44/42

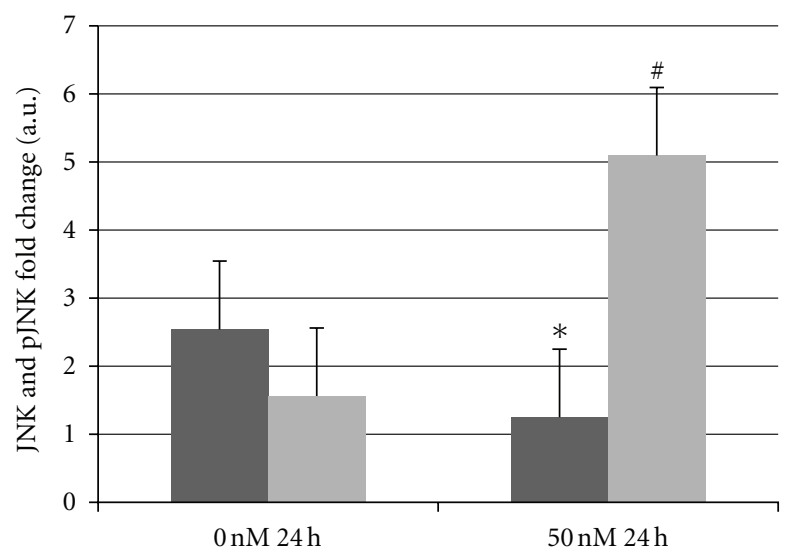

JNK

pJNK

(b)

(c)

FIgure 3: $(\mathrm{a}-\mathrm{c})$ : C2C12 cells were differentiated from myoblasts to myotubes as described in Section 2 . Myotubes were transfected at each concentration of siRNA (SI00906220) (0 and $50 \mathrm{nM}$ ) and collected at the designated time point (24 h). Proteins were collected from these samples and subjected to immunoblot analysis. Data shown are representative of multiple independent experiments $(n=2$ to 4$)$, all analyzed in triplicate $\left({ }^{*} P<0.05,{ }^{*} P<0.1\right)$.

any increase in the basal glucose uptake, we strongly feel that the upregulation of GLUT1 is more of a compensatory mechanism against insulin resistance in $\mathrm{C} 210 \mathrm{c} /-$, since there is no change in GLUT4; the precise underlying mechanism, however, remains to be clarified. This result may prove to be the most crucial, as GLUT4 is the main player in insulinstimulated glucose metabolism. Reports additionally indicate that the activation of $\mathrm{p} 38$ reduces insulin responsiveness [25], so the exact mechanism by which the MAPK pathway is involved in glucose metabolism still remains controversial.

Up-regulation of MyoD and Actin in transfected cells $(\mathrm{C} 210 \mathrm{c} /-)$ suggests a regulatory mechanism by which the myotubes are trying to combat the stressful state of insulin resistance. By aiding with GLUT4-containing vesicle membrane movement and/or fusion, there is considerable evidence that Actin is essential for insulin-regulated glucose transport. Therefore, these cells may be overexpressing Actin acutely in preparation for insulin-stimulated glucose uptake [29].

To confirm the involvement of p38, the p38-specific inhibitor SB203580 was added to C210c/- cells at a concentration of $10 \mu \mathrm{m}$ for $60 \mathrm{~min}[30,31]$. Protein samples were subjected to immunoblot analysis with p38 andphospho-p38 antibodies (Figure 5).

Our results indicate that $\mathrm{C} 210 \mathrm{c} /$ - cells treated with 10 nM SB203580 for 60 min effected the expression of both 


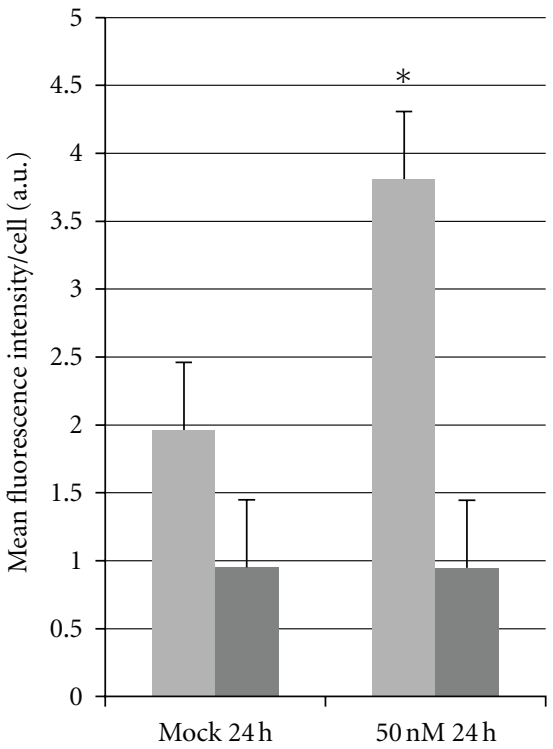

Glut1

Glut4

FIGURE 4: C2C12 cells were seeded onto chamber slides and allowed to differentiate from myoblasts to myotubes as described in the Materials and Methods section. Myotubes were then transfected at each concentration of siRNA (SI00906220) (0 and $50 \mathrm{nM})$ and collected at the designated time point $(24 \mathrm{~h})$. After transfections, cells underwent standard immunofluorescence processing and were imaged. Each sample was compared to negative and positive controls, which were used to quantify the image results $\left({ }^{*} P<0.05\right)$.

p38 $(P=0.1)$ and phospho-p38 $(P=0.08)$ (Figure 5). While not significant, it appears that the inhibitor was able to partially restore the expression of all the proteins tested suggesting its action on the MAPKs. This was further confirmed by the glucose uptake assay performed on C210c/and MAPK-inhibited cells. Results from the assay showed that glucose uptake remained unchanged, confirming that the inhibitors are acting directly on the MAPK proteins and are not affected by changes in Atp10c expression (Figure 6).

\section{Conclusions}

A phenomenon known as phospholipid randomization affects the structure and function of many channels, transporters, and signal transducing proteins and has been implicated in several pathophysiology processes. Thus, maintaining the organization and activity of the lipid bilayer is essential for normal cell function. One class of proteins that performs this action is the flippases or type-IV ATPases [6]. In yeast studies, these proteins cause the translocation of glycerophospholipids, and this movement is necessary for intracellular membrane and protein trafficking [6]. Atp10c is one such phospholipid translocase which encodes for

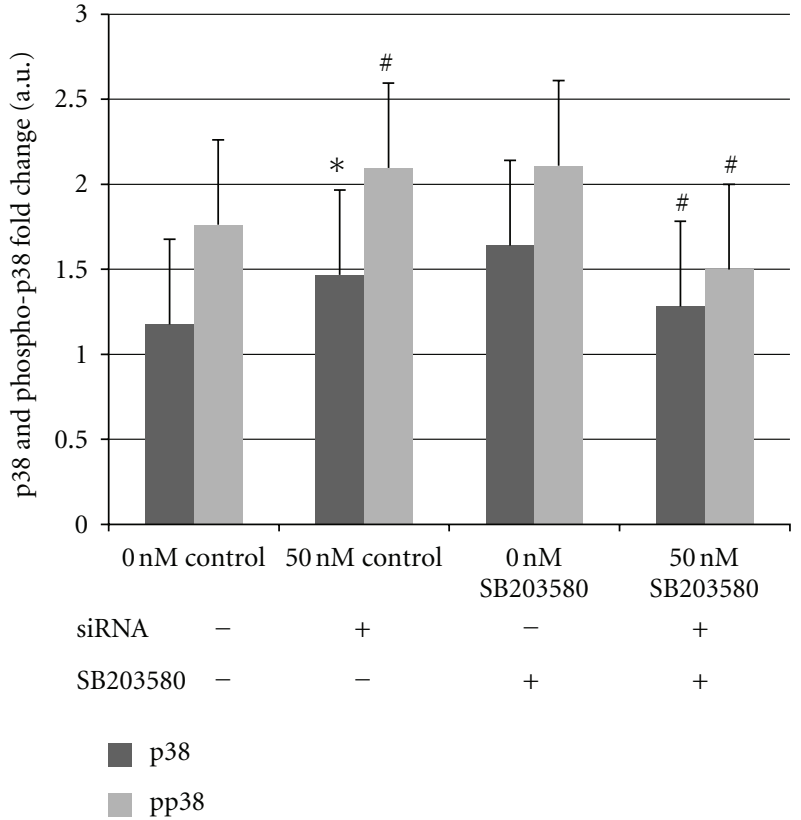

Figure 5: C2C12 cells were differentiated from myoblasts to myotubes as described in the Materials and Methods section. Myotubes were transfected at each concentration of siRNA (SI00906220) (0 and $50 \mathrm{nM})$ at the designated time point $(24 \mathrm{~h})$. Cells were then treated with the inhibitor SB203580 (10 nM) and collected after $60 \mathrm{~min}$. Proteins were collected from these samples and subjected to immunoblot analysis $\left({ }^{*} P<0.05,{ }^{\#} P<0.1\right)$.

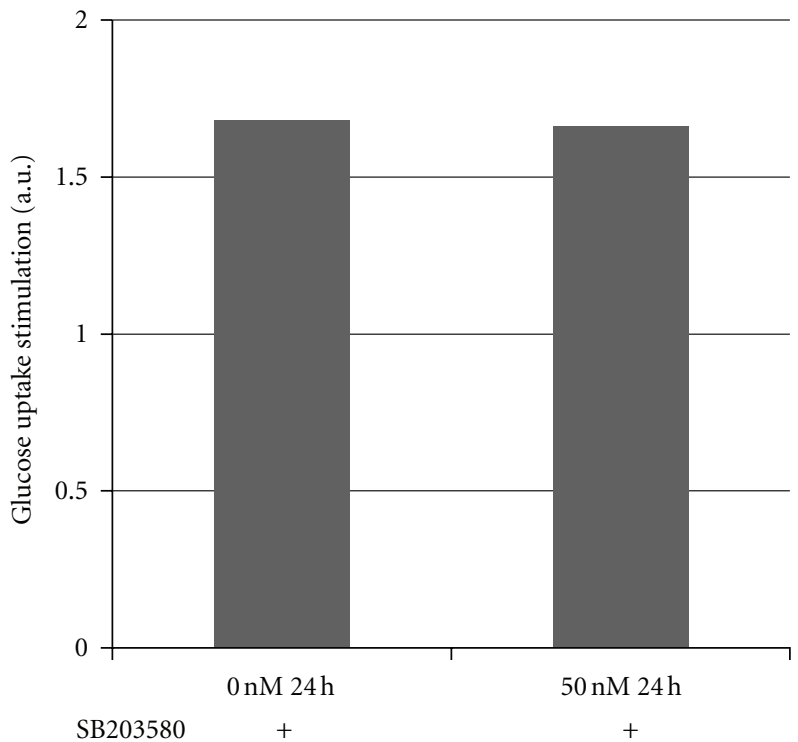

FIgure 6: C2C12 cells were differentiated from myoblasts to myotubes as described in the Materials and Methods section. Myotubes were treated with the inhibitor SB203580 (10 nM) and collected after $60 \mathrm{~min}$. Cells were then stimulated with insulin $(100 \mathrm{nM}, 30 \mathrm{~min})$, and a $2-D O G$ uptake was performed $\left({ }^{*} P<0.05\right.$, $\left.{ }^{\#} P<0.1\right)$. 
a type IV P-type ATPase. Our laboratory has demonstrated that Atp10c heterozygous mice are insulin resistant and have an altered insulin-stimulated response in peripheral tissues. Our obesity mouse model is dietinduced and shows insulin resistance characterized by hyperinsulinemia, hyperglycemia, hyperlipidemia, and obesity in association with glucose intolerance $[2,3,11]$. In fact, a recent publication has sited ATP10C as a potential biomarker for obesity and related metabolic disorders [32].

In the present study, we report, for the first time, a direct correlation between Atp10c mRNA expression levels and glucose metabolism, at least in part via the MAPK pathway in $\mathrm{C} 2 \mathrm{C} 12$ skeletal muscle cells. Our results showed no significant change in the basal glucose uptake suggesting that when Atp10c is silenced using Atp10c-specific siRNA, an acute state of insulin resistance is observed. Conversely, cells are insulin sensitive when normal levels of Atp10c are maintained. The MAPK protein, specifically $\mathrm{p} 38$, is potentially influencing this system by exerting an effect on the glucose transporter proteins. Results from these experiments are interesting and lead to future experiments that will involve other signaling cascades (PI3K), insulin receptors, and substrates as well as other downstream factors, including GLUT4 translocation.

\section{Acknowledgments}

The authors give special thanks to Erin Bartley. Her work as our laboratory assistant is invaluable, and much of this work would not have been possible without her help. They would also like to especially thank the funding agencies: The American Diabetes Association, The University of Tennessee Center of Excellence in Livestock Diseases and Human Health, and the University of Tennessee Obesity Research Center. Additionally, they thank Misty Bailey for her editing assistance.

\section{References}

[1] K. Hayata, K. Sakano, and S. Nishinaka, "Establishment of new highly insulin-sensitive cell lines and screening of compounds to facilitate glucose consumption," Journal of Pharmacological Sciences, vol. 108, no. 3, pp. 348-354, 2008.

[2] M. S. Dhar, C. S. Sommardahl, T. Kirkland et al., "Mice heterozygous for atp10c, a putative amphipath, represent a novel model of obesity and type 2 diabetes," The Journal of Nutrition, vol. 134, no. 4, pp. 799-805, 2004.

[3] M. Dhar, L. S. Webb, L. Smith, L. Hauser, D. Johnson, and D. B. West, "A novel ATPase on mouse chromosome 7 is a candidate gene for increased body fat," Physiol Genomics, vol. 4, no. 1, pp. 93-100, 2000.

[4] M. Meguro, A. Kashiwagi, K. Mitsuya et al., "A novel maternally expressed gene, ATP10C, encodes a putative aminophospholipid translocase associated with Angelman syndrome," Nature Genetics, vol. 28, no. 1, pp. 19-20, 2001.

[5] M. S. Dhar, J. S. Yuan, S. B. Elliott, and C. Sommardahl, "A type IV P-type ATPase affects insulin-mediated glucose uptake in adipose tissue and skeletal muscle in mice," Journal of Nutritional Biochemistry, vol. 17, no. 12, pp. 811-820, 2006.
[6] C. C. Paulusma and R. P. J. Oude Elferink, "Diseases of intramembranous lipid transport," FEBS Letters, vol. 580, no. 23, pp. 5500-5509, 2006.

[7] G. Lenoir and J. C. Holthuis, "The elusive flippases," Current Biology, vol. 14, no. 21, pp. R912-R913, 2004.

[8] N. Kumar and C. S. Dey, "Development of insulin resistance and reversal by thiazolidinediones in $\mathrm{C} 2 \mathrm{C} 12$ skeletal muscle cells," Biochemical Pharmacology, vol. 65, no. 2, pp. 249-257, 2003.

[9] B. Bisht, H. L. Goel, and C. S. Dey, "Focal adhesion kinase regulates insulin resistance in skeletal muscle," Diabetologia, vol. 50, no. 5, pp. 1058-1069, 2007.

[10] S. Roshwalb, S. Gorman, S. Hurst et al., "mRNA expression of canine ATP10C, a P4-type ATPase, is positively associated with body condition score," The Veterinary Journal, vol. 190, no. 1, pp. 174-175, 2011.

[11] A. L. Peretich, M. Cekanova, S. Hurst, S. J. Baek, and M. S. Dhar, "PPAR agonists down regulate the expression of atp10c mRNA during adipogenesis," The Open Obesity Journal, vol. 1, pp. 41-54, 2009.

[12] M. W. Pfaffl, "A new mathematical model for relative quantification in real-time RT-PCR," Nucleic Acids Research, vol. 29, no. 9 , p. e45, 2001.

[13] M. Hance, M. S. Dhar, and H. K. Plummer III, “Tobacco carcinogens stimulate different signaling pathways in breast cancer," Breast Cancer, vol. 1, pp. 25-34, 2008.

[14] M. S. Dhar and H. K. Plummer III, "Protein expression of G-protein inwardly rectifying potassium channels (GIRK) in breast cancer cells," BMC Physiology, vol. 6, article no. 8, 2006.

[15] B. Bisht and C. S. Dey, "Focal Adhesion Kinase contributes to insulin-induced actin reorganization into a mesh harboring glucose transporter-4 in insulin resistant skeletal muscle cells," BMC Cell Biology, vol. 9, article no. 48, 2008.

[16] T. N. Lui, C. W. Tsao, S. Y. Huang, C. H. Chang, and J. T. Cheng, "Activation of imidazoline I2B receptors is linked with AMP kinase pathway to increase glucose uptake in cultured C2C12 cells," Neuroscience Letters, vol. 474, no. 3, pp. 144-147, 2010.

[17] T. Nedachi and M. Kanzaki, "Regulation of glucose transporters by insulin and extracellular glucose in $\mathrm{C} 2 \mathrm{C} 12$ myotubes," American Journal of Physiology, vol. 291, no. 4, pp. E817-E828, 2006.

[18] T. Shimokawa, M. Kato, O. Ezaki, and S. Hashimoto, "Transcriptional regulation of muscle-specific genes during myoblast differentiation," Biochemical and Biophysical Research Communications, vol. 246, no. 1, pp. 287-292, 1998.

[19] E. B. Katz, R. Burcelin, T. S. Tsao, A. E. Stenbit, and M. J. Charron, "The metabolic consequences of altered glucose transporter expression in transgenic mice," Journal of Molecular Medicine, vol. 74, no. 11, pp. 639-652, 1996.

[20] R. T. Watson, M. Kanzaki, and J. E. Pessin, "Regulated membrane trafficking of the insulin-responsive glucose transporter 4 in adipocytes," Endocrine Reviews, vol. 25, no. 2, pp. 177-204, 2004.

[21] G. I. Shulman, "Cellular mechanisms of insulin resistance," Journal of Clinical Investigation, vol. 106, no. 2, pp. 171-176, 2000.

[22] R. T. Watson and J. E. Pessin, "Intracellular organization of insulin signaling and GLUT4 translocation," Recent Progress in Hormone Research, vol. 56, pp. 175-193, 2001.

[23] P. R. Shepherd and B. B. Kahn, "Glucose transporters and insulin action: implications for insulin resistance and diabetes mellitus," New England Journal of Medicine, vol. 341, no. 4, pp. 248-257, 1999. 
[24] H. Wallberg-Henriksson and J. R. Zierath, "GLUT4: a key player regulating glucose homeostasis? Insights from transgenic and knockout mice," Molecular Membrane Biology, vol. 18, no. 3, pp. 205-211, 2001.

[25] M. Fujishiro, Y. Gotob, H. Katagiri et al., "MKK6/3 and p38 MAPK pathway activation is not necessary for insulin-induced glucose uptake but regulates glucose transporter expression," Journal of Biological Chemistry, vol. 276, no. 23, pp. 1980019806, 2001.

[26] N. Kumar and C. S. Dey, "Gliclazide increases insulin receptor tyrosine phosphorylation but not p38 phosphorylation in insulin-resistant skeletal muscle cells," Journal of Experimental Biology, vol. 205, no. 23, pp. 3739-3746, 2002.

[27] N. Wijesekara, F. S. L. Thong, C. N. Antonescu, and A. Klip, "Diverse signals regulate glucose uptake into skeletal muscle," Canadian Journal of Diabetes, vol. 30, no. 1, pp. 80-88, 2006.

[28] M. Ariga, T. Nedachi, H. Katagiri, and M. Kanzaki, "Functional role of sortilin in myogenesis and development of insulin-responsive glucose transport system in $\mathrm{C} 2 \mathrm{C} 12$ myocytes," Journal of Biological Chemistry, vol. 283, no. 15, pp. 10208-10220, 2008.

[29] A. M. McCarthy, K. O. Spisak, J. T. Brozinick, and J. S. Elmendorf, "Loss of cortical actin filaments in insulin-resistant skeletal muscle cells impairs GLUT4 vesicle trafficking and glucose transport," American Journal of Physiology, vol. 291, no. 5, pp. C860-C868, 2006.

[30] M. Koistinaho and J. Koistinaho, "Role of p38 and p44/42 mitogen-activated protein kinases in microglia," Glia, vol. 40, no. 2, pp. 175-183, 2002.

[31] H. Wang, Q. Xu, F. Xiao, Y. Jiang, and Z. Wu, "Involvement of the $\mathrm{p} 38$ mitogen-activated protein kinase $\alpha, \beta$, and $\gamma$ isoforms in myogenic differentiation," Molecular Biology of the Cell, vol. 19, no. 4, pp. 1519-1528, 2008.

[32] F. I. Milagro, J. Campión, P. Cordero et al., "A dual epigenomic approach for the search of obesity biomarkers: DNA methylation in relation to diet-induced weight loss," The FASEB Journal, vol. 25, no. 4, pp. 1378-1389, 2011. 


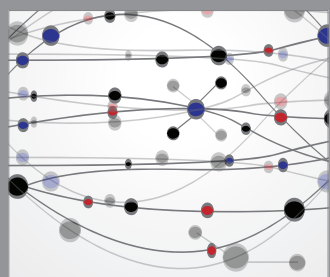

The Scientific World Journal
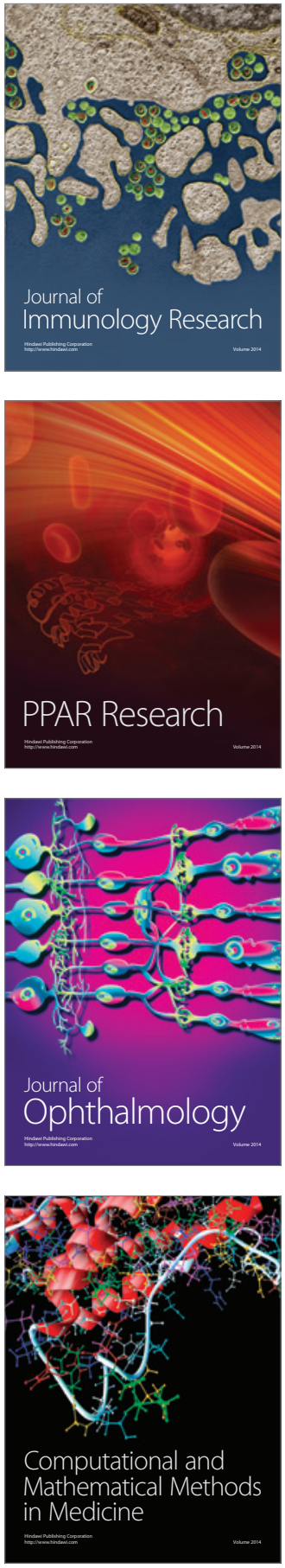

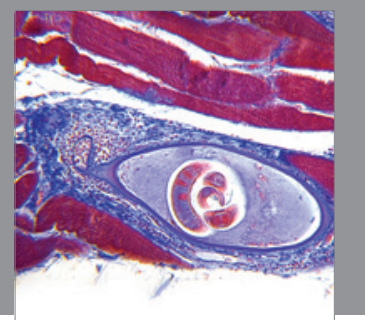

Gastroenterology

Research and Practice
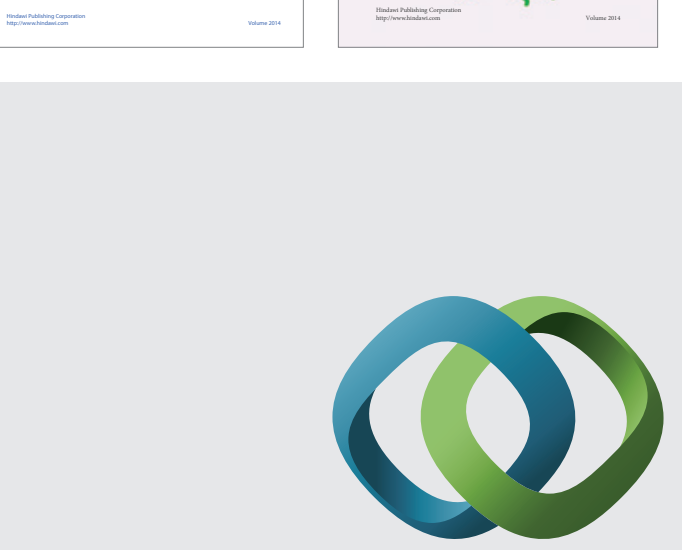

\section{Hindawi}

Submit your manuscripts at

http://www.hindawi.com
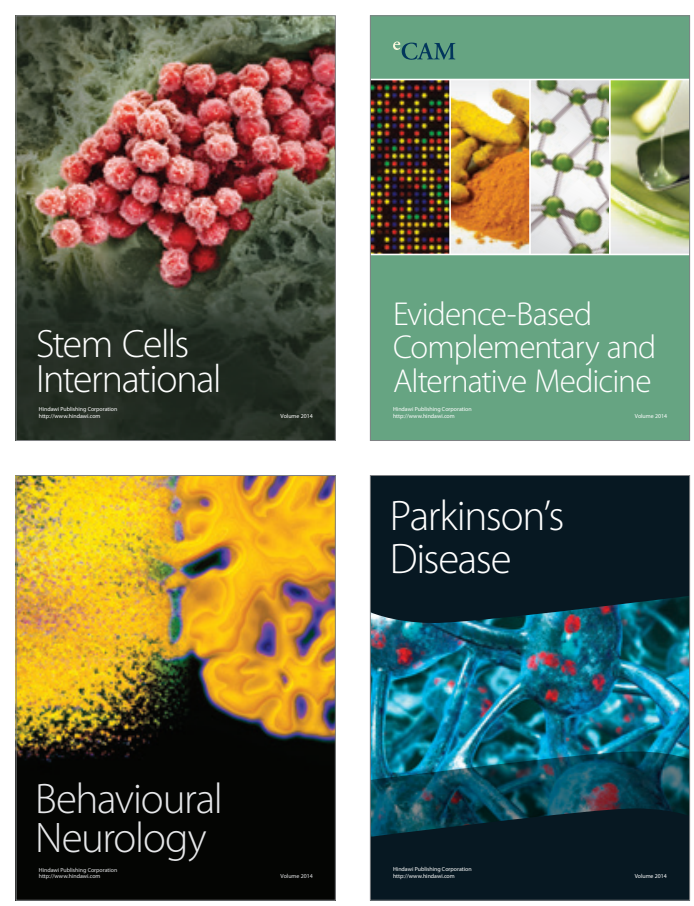

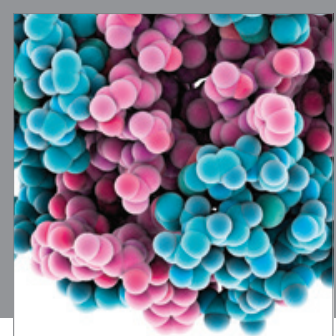

Journal of
Diabetes Research

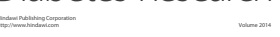

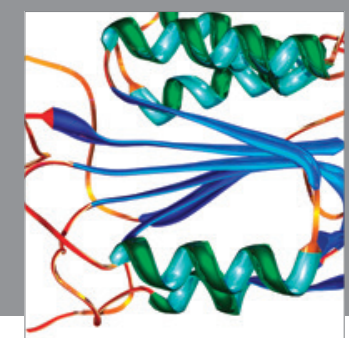

Disease Markers
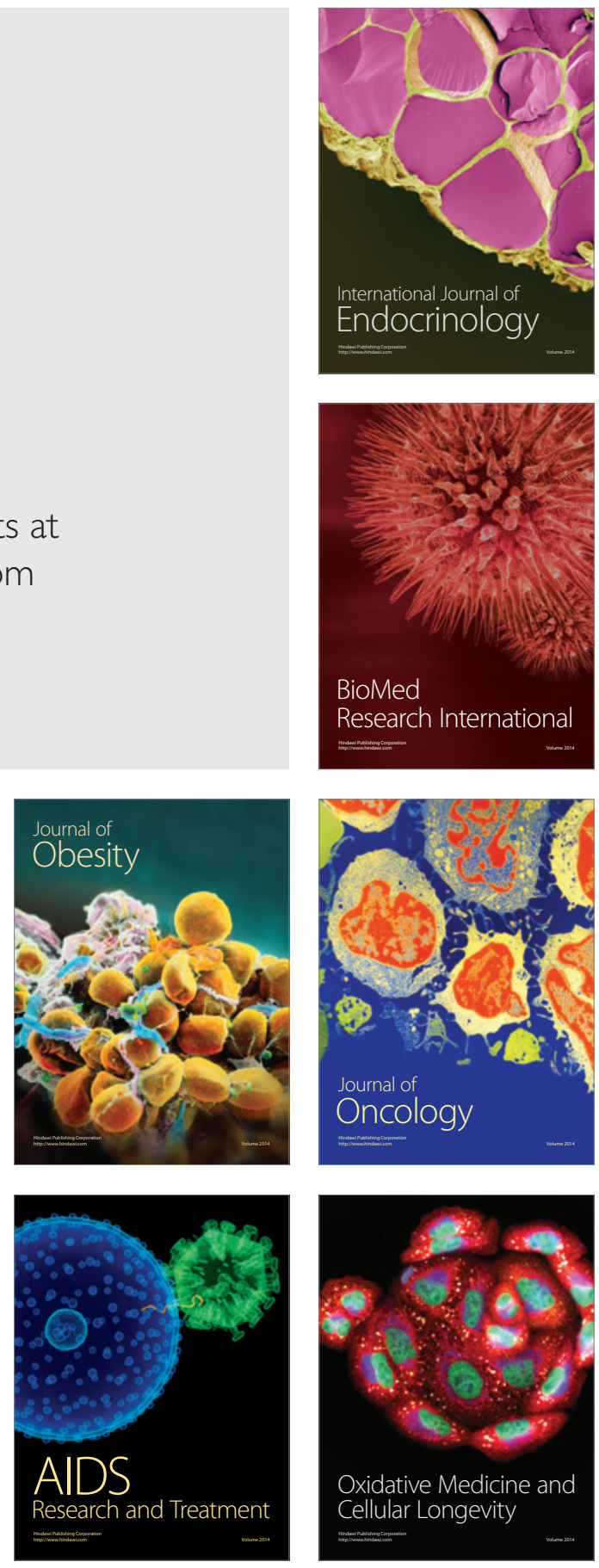